\title{
МОДЕЛИРОВАНИЕ СТРУКТУРЫ И ОБЪЕМОВ ПРОИЗВОДСТВА ЛЕСНОГО СЕКТОРА В ЛЕСНОМ ПЛАНИРОВАНИИ
}

\author{
(C) 2019 Третьяков Александр Георгиевич \\ кандидат экономических наук, доцент кафедры экономики и менеджмента \\ Омский региональный институт, Россия, Омск
}

В данной статье рассматривается модель формирования структуры и объемов производства лесного сектора, учитывающая экономическую доступность лесных ресурсов. Эта модель основана на комбинированном подходе обоснования объемов производства и ассортимента продукции лесного комплекса, и представляет собой задачу целочисленного линейного программирования. Раскрыто экономическое содержание результатов решения данной модели, и обозначена их роль в лесном планировании.

Ключевые слова: лесные ресурсы, заготовка и переработка древесины, лесное планирование, модель, экономическая доступность лесов, линейное программирование.

Одной из главных задач лесного планирования является обоснование объемов производства и ассортимента продукции лесного комплекса, что обеспечивает формирование стоимостных выходных показателей плана по использованию лесов. Актуальность данной проблемы обосновывается задачей интенсификации использования и воспроизводства лесов, сформированной в «Основах государственной политики в области использования, охраны, защиты и воспроизводства лесов в Российской Федерации на период до 2030 года» [1], которая предусматривает рост объемов лесопользования с единицы лесной площади и оптимизацию его структуры.

Решение проблемы обоснования объемов производства возможно при использовании трех принципиально отличных друг от друга подходов (рыночного, ресурсного, комбинированного), анализ которых был осуществлен автором ранее [9]. Данные подходы в определяющей мере отличаются выбором фундамента для планирования показателей использования лесов, так рыночный подход основывается на характеристиках спроса на лесную продукцию, а ресурсный рассматривает существующее предложение древесного сырья исходя из характеристик лесосечного фонда. Наиболее перспективно использование комбинированного подхода формирования объемов и структуры производства лесного сектора, который основывается на осуществлении оценки экономической доступности лесных ресурсов, включая сопоставление спроса на лесные ресурсы с их предложением.
Значимость показателя экономической доступности значительно возросла в связи с «Основами государственной политики ...» [1], в которых предусматривается «переход к определению расчетной лесосеки с учетом экономической доступности лесов и их деления по целевому назначению, а также уровня развития транспортной инфраструктуры, товарной и породно-возрастной структуры насаждений», однако по настоящее время не существует нормативных документов, регламентирующих расчет данного показателя.

В научных исследованиях существуют различные подходы к определению критерия экономической доступности, например в отдельных работах он рассматривался как обеспечение нормативного уровня рентабельности при использовании современного организационнотехнического уровня освоения рассматриваемых ресурсов [7]. Однако, данный подход не учитывает современный экономический механизм аренды лесов, когда лесопользователь (арендатор участка леса) осуществляет затраты на воспроизводство, охрану и защиту лесов за свой счет. Поэтому автор оценивает экономическую доступность лесных ресурсов на основе критерия, который предусматривает равенство или превышение значения лесной ренты от заготовки древесного сырья над величиной нормативной стоимости воспроизводства, охраны и защиты лесов $[4,5,9,11]$. Лесная рента рассматривается как чистый доход формируемый в момент использования лесных ресурсов и определяемый как разница между рыночной ценой 
конечной лесопродукции и затратами на ее производство при учете обеспечения нормативной прибыли предпринимателя (при расчете затрат на производство лесопродукции платежи за лесные ресурсы не включаются). Данной трактовки этого показателя придерживается большинство исследователей в области лесной экономики [5, $6,10]$.

Использование показателя экономической доступности лесных ресурсов, отражающий степень эффективности их освоения, позволяет разрабатывать лесные планы, обеспечивающие оптимальную структуру лесозаготовок. Однако формирование данной структуры требует учета множества производственных и экономических факторов, которые часто выступают в качестве ограничений тех или иных управленческих решений и которые следует включать в расчеты. Это предопределяет необходимость формирования математической модели расчета объемов и структуры лесозаготовок, учитывающей как получение максимального эффекта, так и необходимость выполнения различных ресурсных и экономических ограничений. Данная статья посвящена формированию такой математической модели, решение которой на конкретных данных позволит обеспечить формирование оптимальных лесных планов с позиций как удовлетворения потребности народного хозяйства в лесоматериалах, так и в максимизации экономического эффекта от заготовки древесины.

Задачу определения объемов и структуры лесозаготовок предлагается решить методами оптимизации, практика использования которых широко распространена в лесном планировании $[2,3,8]$, однако они были сформированы с учетом предшествующей практики хозяйственной деятельности, не учитывающей использование арендных отношений в лесном секторе.

Оптимальная производственная структура лесного сектора, которая является главным выходным показателем лесного планирования, достигается тогда, когда:

1) лесосырьевые ресурсы распределены по существующим и создаваемым производствам, а также по поставкам сортиментов за пределы региона, таким образом, чтобы формируемая оценка экономической доступности лесных ресурсов была максимальным;

2) вновь вводимые производственные мощности ориентированы на полное комплексное использование сырья через применение ин- новационных безотходных технологий;

3) модернизация существующих производств и размещение новых производств обеспечивают баланс экономических, социальных и экологических целей;

Также в лесном плане должно быть предусмотрено формирование оптимальной лесной инфраструктуры (лесовозных дорог, складское хозяйство), формирующей необходимы условия для сырьевого обеспечения перерабатывающих предприятий.

Названные выше условия, создающие оптимальную производственную структуру, реализуются решением задачи линейного целочисленного программирования с целевой функцией максимизации чистого дохода, определяемого как разница между валовым доходом от реализации лесопродукции и совокупными затратами на воспроизводство, охрану и защиту лесов и производство лесопродукции с учетом нормативной прибыли на капитал (в затраты на производство не входят платежи за лесные ресурсы).

Для решения оптимизационной задачи для многолесного региона, в котором потенциальный объем заготовки древесины превышает объем ее потребления, принимаем:

$\mathrm{d}$ - индекс вида первичного сырья (породноразмерная группа),

В рамках рассматриваемой задачи, используются следующие значения данного индекса: 1 - пиловочник, 2 - балансы, 3- фанерный кряж, 4 - сортименты круглого леса для производства плит и биоэнергии, 5 - сортименты круглого леса для потребления в круглом виде (столбы, рудстойка и др.).

d" - индекс вида вторичного сырья, представленного образующимися отходами,

i - индекс вида продукции лесного комплекса (1- пиломатериалы, 2 - целлюлоза, 3 - фанеpa, 4 - сортименты круглого леса для потребления в круглом виде (столбы, рудстойка и др.), 5 - плиты, пеллеты,

j - индекс выдела,

е - индекс предприятия (производства, места отгрузки древесного сырья),

e"" - индекс места отгрузки древесного сырья в другие регионы,

$\mathrm{n}$ - горизонт планирования (10 лет), лет

$\mathrm{n}_{\mathrm{e}}$ - срок функционирования нового е-го предприятия, в рамкам горизонта планирования, лет

1 - индекс новой лесовозной дороги, 
$\mathrm{n}_{1}$ - срок функционирования новой 1-ой лесовозной дороги в рамкам горизонта планирования, лет

$\mathrm{k}$ - индекс варианта типовой мощности новых производств,

$\mathrm{r}$-индекс участка леса, по которому устанавливается допустимый объем пользования,

$\mathrm{x}_{\mathrm{djei}}$ - объем поставки d-го вида сырья из j-ого выдела на е-ое предприятие для производства і-ой продукции,

$\mathrm{x}_{\text {dje" }}$ - объем поставки d-го вида сырья из j-ого выдела на е"”-ое место отгрузки для поставки в другие регионы,

$\mathrm{x}_{\mathrm{d} " \mathrm{ee} " \mathrm{i}}$ - объем поставки d"-го вида вторичного сырья из е-ого предприятия в е"-ое предприятие для производства і-ой продукции,

$\mathrm{X}_{\mathrm{j}}$ - целочисленная переменная, отражающая проведение лесозаготовок в ј-ом выделе в рамках рассматриваемого периода времени,

$\mathrm{XD}_{1}$ - целочисленная переменная, отражающая строительство 1-ой лесовозной дороги в рамках рассматриваемого периода времени,

$\mathrm{Y}_{\mathrm{e} " \mathrm{i}}$ - целочисленная переменная, отражающая объемы производства на новом е"-ом предприятии при производстве i-ой продукции, ед. продукции (переменная может принимать значения равные 0 , а также типовые значения мощности «новых» производств),

$\mathrm{V}_{\mathrm{j}}$ - эксплуатационный запас $\mathrm{j}$-ого выдела, предназначенный для рубки в рамкам рассматриваемого периода времени,

$y_{d j}$ - выход d-го вида сырья в ј-ом выделе,\% (например, у1ј выход пиловочника в ј-ом выделе)

$\mathrm{D}_{\mathrm{r}}$ - объем допустимого пользования в $\mathrm{r}$-ом участке леса (r-ом лесничестве), м3/год,

$\mathrm{M}_{\mathrm{ki}}$ - величина типовой $\mathrm{k}-$ ой мощности для i-ого производства,

$\gamma_{\mathrm{d} 1 \mathrm{~d} 2 \mathrm{i}}$ - норма образования $\mathrm{d}_{2}$-го вида вторичного сырья при переработке $\mathrm{d}_{1}$-го вида первичного сырья в і-ом производстве, ед. продукции $/ \mathrm{M}^{3}$,

$\mathrm{Q}_{\mathrm{i}}$ - ограничение по объему спроса для производства i-ого вида продукции, ед. продукции/ год,

$Q^{\prime}{ }_{d}$ - ограничение по объему спроса для поставок d-ого вида первичного сырья из региона, $\mathrm{M}^{3} /$ год,

$\mathrm{Z}_{\mathrm{dei}}$-предельная цена d-ого вида сырья на е-том предприятии при производстве i-ой продукции

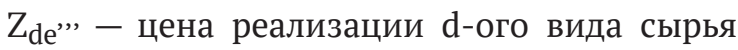

вывозимого из региона в е"'-ом пункте отгрузки.

$t_{\text {je }}$ - затраты на транспортировку древесного сырья для ј-ого выдела на е-ое предприятие (место отгрузки), руб/м³,

$\mathrm{f}_{\mathrm{j}}$ - нормативная стоимость воспроизводства, охраны и защиты лесов для ј-ого выдела, руб $/ \mathrm{M}^{3}$,

$\mathrm{C}_{\mathrm{j}}$ - нормативные затраты на заготовку древесины для ј-ого выдела, руб/м³,

$\mathrm{p}_{\mathrm{j}}$ - нормативная прибыль на заготовку древесины для ј-ого выдела, руб/м³,

$\mathrm{Z}_{\mathrm{d} " \mathrm{e}} \mathrm{i}$-предельная цена $\mathrm{d}$ "-ого вида вторичного сырья на е"-том предприятии при производстве і-ой продукции, руб/ед. сырья.

$t_{\text {eе" }}$ - затраты на транспортировку вторичного сырья из е-ого предприятия в е"-ое предприятие, руб/ед. сырья,

$\mathrm{C}_{\mathrm{d} \text { e }}$ - нормативные затраты производства $\mathrm{d}$ "-ого вида вторичного сырья на е-том предприятии, руб/ед. сырья,

$\mathrm{p}_{\mathrm{d}} \mathrm{e}-$ нормативная прибыль производства вторичного сырья на е-том предприятии, руб/ед. сырья,

$\operatorname{Pr}_{d e i}$ - цена реализации i-ой лесопродукции произведенной на е-ом предприятии из d-ого вида сырья, руб/ед. продукции,

$\mathrm{q}_{\text {dei }}$ - нормативные затраты производства i-ой лесопродукции на е-ом предприятии из d-ого вида сырья, руб/ед. продукции,

$\mathrm{P}_{\text {dei }}$ - нормативная прибыль производства i-ой лесопродукции на е-ом предприятии из d-ого вида сырья, руб/ед. продукции,

$\mathrm{m}_{\mathrm{dei}}$ - норма расхода $\mathrm{d}$-ого вида сырья производства i-ой лесопродукции на е-ом предприятии, $\mathrm{M}^{3} /$ ед. продукции,

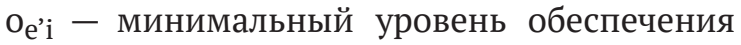
сырьем, заготовленным в регионе, производственных мощностей «новых» е'-ых предприятий производящих і-ую лесопродукцию,\%,

$\mathrm{W}_{\mathrm{ei}}$ - производственная мощность е-ого существующего предприятия по i-ому виду продукции, ед. продукции,

$\mathrm{K}_{\mathrm{di}}$ - удельные капитальные вложения на производство i-ой продукции из d-го вида сырья,

КK - ограничение по возможностям привлечения инвестиций в развитие перерабатывающих производств в регионе в рамкам рассматриваемого горизонта планирования, руб.

ККК - ограничение по возможностям привлечения инвестиций в строительство лесовозных дорог производств в регионе в рамкам рассматриваемого горизонта планирования, руб. 

руб.

$\mathrm{KD}_{1}$ - затраты на строительство 1-ой дороги, $\mathrm{VV}_{\operatorname{maxl}}$ и $\mathrm{VV}_{\operatorname{minl}}$ - максимальный и минимально экономически обоснованный уровень грузооборота по 1-ой дороге, $\mathrm{M}^{3}$.

При принятых выше параметрах целевая функция для оптимизации структуры потребления древесины в границах ее экономически доступных ресурсов будет представлена следующим образом, как сумма трех эффектов - от древесины используемой в регионе, от древесины вывозимой в другие регионы и от использования древесных отходов.

$$
\begin{aligned}
& \sum_{d j e i} x_{d j e i}\left[Z_{d e i}-t_{j e}-f_{i}-C_{j}-p_{j}\right] \\
& +\sum_{d j e^{\prime \prime \prime}} x_{d j e^{\prime \prime \prime}}\left[Z_{d e^{\prime \prime \prime}}-t_{j e^{\prime \prime \prime}}-f_{i}-C_{j}-p_{j}\right] \\
& +\sum_{e^{\prime \prime} i} x_{d^{\prime \prime} e e^{\prime \prime} i}\left[Z_{d^{\prime \prime} e^{\prime \prime}}-t_{e e^{\prime \prime}}-C_{d^{\prime \prime} e}-p_{d^{\prime \prime} e}\right] \rightarrow \max
\end{aligned}
$$

где предельная цена сырья определяется на основе следующей формулы

$$
Z_{d e i}=\frac{P r_{d e i}-q_{d e i}-P_{d e i}}{m_{d e i}}
$$

Предельная цена для вторичного сырья $\left(Z_{\mathrm{d} " \mathrm{e}} \mathrm{i}\right)$ определяется по аналогичной формуле, цены на древесину вывозимую из региона $\left(\mathrm{Z}_{\mathrm{de}}\right.$ "') формируются на основе рыночного мониторинга.

При выполнении оптимизационных расчетов существуют следующие ограничения.

Ограничения по ресурсам сырья на уровне выдела:

1. Лесопильные предприятия потребляют только пиловочник - объем поставок пиловочника на лесопильные предприятия региона и за пределы региона из і-ого выдела не превышает потенциальный запас пиловочника в выделе.

$$
\sum_{e} x_{1 j e 1}+\sum_{e \prime \prime \prime} x_{1 j e \prime \prime \prime} \leq V_{j} * \frac{y_{1 j}}{100}
$$

2. Фанерные предприятия потребляют березовый или сосновый кряж

$$
\sum_{e} x_{3 j e 3}+\sum_{e \prime \prime \prime} x_{3 j e ! \prime \prime} \leq V_{j} * \frac{y_{3 j}}{100}
$$

3. Целлюлозные предприятия могут потреблять пиловочник, фанерный кряж и балансы

$$
\begin{gathered}
\sum_{e} x_{1 j e 2}+\sum_{e} x_{2 j e 2}+\sum_{e} x_{3 j e 2}+\sum_{e^{\prime \prime}} x_{2 j e^{\prime \prime \prime}} \leq V_{j} * \frac{y_{1 j}+y_{2 j}+y_{3 j}}{100} \\
-\sum_{e} x_{1 j e 1}-\sum_{e} x_{3 j e 3}-\sum_{e^{\prime \prime \prime}} x_{1 j e^{\prime \prime \prime}}-\sum_{e^{\prime \prime \prime}} x_{3 j e^{\prime \prime \prime}}
\end{gathered}
$$

4. Объемы поставки круглых материалов в необработанном виде.

$$
\sum_{e} x_{5 j e 5}+\sum_{e \prime \prime \prime} x_{5 j e \prime \prime \prime} \leq V_{j} * \frac{y_{5 j}}{100}
$$

5. Производство плит и энергии не имеют ограничений по качеству сырья.

В выделах, где проводится рубка, вся древесина, за исключением направляемой на лесопильные, фанерные и целлюлозные предприятия, а также отправляемой в другие регионы или используемой в круглом виде, может быть утилизирована для производства плит и энергии.

$$
\begin{aligned}
& \sum_{e} x_{1 j e 2}+\sum_{e} x_{2 j e 2}+\sum_{e} x_{3 j e 2}+\sum_{e} x_{1 j e 1}+\sum_{e} x_{3 j e 3} \\
& +\sum_{e} x_{5 j e 5}+\sum_{d e^{\prime \prime}} x_{d j e^{\prime \prime \prime}}+\sum_{d e} x_{d j e 4}=X_{j} * V_{j^{\prime}}
\end{aligned}
$$

Ограничения по формированию вторичных отходов.

6. Потенциал формирования вторичных отходов на е-ом предприятии равен объему использования этих отходов

$$
\sum_{e^{\prime i}} x_{d " e e " i}=\sum_{d j i}\left(x_{d j e i *} \gamma_{d d^{\prime \prime} i}\right)
$$

Ограничения объемов потребления сырья на уровне предприятий.

7. Лесопильное производство

для существующих предприятий - объем поставок сырья на предприятие не может превышать его производственной мощности

$$
\sum_{j} x_{1 j e 1} \leq n * W_{e 1} * m_{1 e 1}
$$

для новых предприятий

$$
\sum_{j} x_{1 j e^{\prime} 1} \leq n_{e^{\prime}} * Y_{e^{\prime} 1} * m_{1 e^{\prime} 1}
$$

Обеспечение загрузки производственных мощностей древесным сырьем, заготовленным в регионе - объем поставок древесного сырья на новое предприятие должен быть не ниже зна- 
чения обеспечивающего минимальный уровень их загрузки.

$$
\sum_{j} x_{1 j e^{\prime} 1} \geq n_{e^{\prime}} * Y_{e^{\prime} 1} * m_{1 e^{\prime} 1} * \frac{o_{e^{\prime} 1}}{100}
$$

Аналогичные неравенства обеспечения минимального объема уровня загрузки формируются для всех новых предприятий производящих другие виды продукции.

8. Фанерное производство

для существующих предприятий

$$
\sum_{j} x_{3 j e 3} \leq n * W_{e 3} * m_{3 e 3}
$$

для новых предприятий

$\sum_{j} x_{3 j e^{\prime} 3} \leq n_{e^{\prime}} * Y_{e^{\prime} 3} * m_{3 e^{\prime} 3}$

9. Целлюлозные предприятия для существующих предприятий

$\sum_{d} \frac{\sum_{j} x_{d j e 2}}{m_{d e 2}}+\sum_{d^{\prime \prime}} \frac{\sum_{e^{\prime \prime}} x_{d^{\prime \prime} e " e 2}}{m_{d^{\prime \prime} e 2}} \leq n * W_{e 2}$

для новых предприятий

$\sum_{d} \frac{\sum_{j} x_{d j e^{\prime} 2}}{m_{d e^{\prime} 2}}+\sum_{d^{\prime \prime}} \frac{\sum_{e^{\prime \prime}} x_{d^{\prime \prime} e^{\prime \prime} e^{\prime} 2}}{m_{d^{\prime \prime} e^{\prime} 2}} \leq n_{\mathrm{e}^{\prime}} * Y_{e^{\prime} 2}$

где $\mathrm{d}=1,2,3$

10. Производство плит и энергии для существующих предприятий

$\sum_{d} \frac{\sum_{j} x_{d j e 4}}{m_{d e 4}}+\sum_{d^{\prime \prime}} \frac{\sum_{e^{n}} x_{d e e 4}}{m_{d^{\prime \prime} e}} \leq n * W_{e 4}$

для новых предприятий

$$
\sum_{d} \frac{\sum_{j} x_{d j e^{\prime} 4}}{m_{d e^{\prime} 4}}+\sum_{d^{\prime \prime}} \frac{\sum_{e^{\prime \prime}} x_{d^{\prime \prime} e^{\prime \prime} e^{\prime} 4}}{m_{d^{\prime \prime} e^{\prime} 4}} \leq n_{\mathrm{e}^{\prime}} * Y_{e^{\prime} 4}
$$

11. Объемы поставки круглых материалов в необработанном виде задаются директивно в разрезе мест отгрузки.

$$
\sum_{j} x_{4 j e 4}=n * W_{e 4}
$$

Ограничения по объему спроса на лесопродукцию в регионе

Объем производства продукции не должен превышать потребностей рынка:

$$
\sum_{e d} \frac{\sum_{j} x_{d j e^{\prime} i}}{m_{d e^{\prime} i}}+\sum_{e d^{\prime \prime}} \frac{\sum_{e^{n}} x_{d^{\prime \prime} e^{\prime \prime} e^{\prime} i}}{m_{d^{\prime \prime} e^{\prime} i}} \leq n * Q_{i}
$$

При формировании данного ограничения не учитываются объемы поставки древесины в места отгрузки сортиментов (e"'), предназначенные для вывоза в другие регионы.

Ограничения по объему спроса на поставки круглого леса из региона

Объем поставок из региона круглых лесоматериалов не должен превышать потенциал спроса на них.

$$
\sum_{e^{\prime \prime \prime} j} x_{d j e ! \prime \prime} \leq n * Q_{d}^{\prime}
$$

Ограничение по допустимому объему пользования

Данное ограничение устанавливается для r-ого участка леса (лесничества) как для всего участка, так и для хвойного хозяйства.

$$
\sum_{d j e i} x_{d j e i}+\sum_{d j e \prime \prime \prime} x_{d j e \prime \prime \prime} \leq n * D_{r}
$$

Индексация выделов производится таким образом, чтобы можно выделить совокупности выделов, относящиеся:

- к r-ому участку леса,

- к хвойному хозяйству r-ого участка леса,

- к 1-ой новой лесовозной дороге (для участков не примыкающих к существующим лесным дорогам).

Ограничение по объемам капитальных вложений в переработку древесины

Объем инвестиций на введение новых производственных мощностей не должен превышать их возможных ресурсов из разных источников финансирования

$$
\sum_{d i}\left(\sum_{e^{\prime}} \frac{\sum_{j} x_{d j e^{\prime} i}+\sum_{e} x_{d^{\prime} e e^{\prime} i}}{n_{e^{\prime}}} * K_{d i}\right) \leq \mathrm{KК}
$$

Ограничение по объемам вывозки древесины по строящимся лесовозным дорогам

Грузооборот по новым строящимся лесовозным дорогам должен превышать минимальный уровень. Рубка в выделах, не примыкающих к существующей сети лесовозных дорог, может осуществляться только в том случае, если строиться новая лесовозная дорога, по которой может осуществляться вывозка древесины с выдела.

$X D_{l} * V V_{\text {minl }} * n_{l} \leq \sum_{j} X_{j} * V_{J} \leq X D_{l} * V V_{\text {maxl }} * n_{l}$ 
Ограничение по объемам затрат на строительство лесовозных дорог

$$
\sum_{l} X D_{l} * K D_{l} \leq K K K
$$

\section{Ограничения на значения величин пере- менных}

$$
\begin{aligned}
& x_{d j e i} \geq 0 \\
& x_{d j e^{\prime \prime \prime}} \geq 0 \\
& x_{d^{\prime \prime} e e i} \geq 0 \\
& X_{j}=\{0,1\} \\
& X D_{l}=\{0,1\} \\
& Y_{e^{\prime} i}=\left\{0, M_{1 i}, M_{2 i}, M_{3 i} \ldots\right\}
\end{aligned}
$$

Таким образом, выходной информацией при решении рассматриваемой оптимизационной задачи будут:

- перечень выделов, поступающих в рубку в рамках планового периода,

- объемы поставки лесных ресурсов различных породно-размерных групп из выделов в существующие и новые перерабатывающие производствах на территории региона,
- объемы поставки круглого леса за пределы региона,

- объемы поставки вторичных ресурсов между перерабатывающими предприятиями,

- перечень «новых» перерабатывающих производств в разрезе их видов и производственных мощностей,

- перечень строящихся лесовозных дорог.

Оптимизация распределения ресурсов древесины позволяет:

1. Исключить из сферы освоения экономически недоступные ресурсы.

2. Обеспечить сырьем потребителей, не превышая при этом существующих и вводимых мощностей.

3. Максимизировать лесной доход на базе баланса интересов государства и бизнеса.

Практическое решение предложенной оптимизационной задачи, которая может составлять основу разрабатываемых лесных планов региона, возможно лишь при наличии информации, представленной оценкой экономической доступности лесных ресурсов региона, чему препятствует отсутствие достоверных лесоучетных материалов, а также информационных баз, требующихся для выполнения расчетов.

\section{Библиографический список}

1. Основы государственной политики в области использования, охраны, защиты и воспроизводства лесов в Российской Федерации на период до 2030 года. Утверждены распоряжением Правительства Российской Федерации от 26 сентября 2013 г. № 1724-р

2. Воевода И.Н. Территориальные отраслевые комплексы: на примере лесной промышленности и лесного хозяйства / И.Н. Воевода, А.П. Петров - Новосибирск: Наука, Сибирское отделение.-1987. - 284 с

3. Коробов П.Н. Математическое программирование и моделирование экономических процессов. - М.: ДНК. 2006. -376 c.

4. Майоров, И.Г. Экономическая доступность лесных ресурсов и транспортная доступность /И.Г. Майоров, А.Г. Третьяков// Экономические науки. - 2014. - № 10 (119). - С. 24-28.

5. Петров, А.П. Рентные платежи - действенный механизм повышения доходности лесопользования / А.П. Петров // «Использование и охрана природных ресурсов в России» - 2002. - № 3. - С. 82-84

6. Починков, С.В. Методика рентной оценки древесных ресурсов леса / С. В. Починков // «Лесное хозяйство»2004. - № 3. - С. 8-13.

7. Пфаиенштиль, И.В. Принципы определения эколого-экономической доступности лесных ресурсов Красноярского края. Диссертация на соискание ученой степени кандидата сельскохозяйственных наук - Красноярск, 2002-127 с.

8. Соколов А.П. Методика принятия решений по оптимизации лесозаготовительных планов/ А.П. Соколов, Ю.Ю. Герасимов // Научный журнал КубГАУ - 2011. -№ 69(05) Электронный журнал http://ej.kubagro. $\mathrm{ru} / 2011 / 05 / \mathrm{pdf} / 29 \mathrm{pdf}$

9. Третьяков, А.Г. Территориальные программы развития лесного сектора: показатели и сбалансированность интересов /А.Г. Третьяков// Известия Санкт-Петербургской лесотехнической академии. - 2015.- № 210.С. 259-273.

10. Эйсмонт, О.А. Оценка лесной ренты и эффективность повышения рентных платежей в России / О.А. Эйсмонт, А.П. Петров, А. В. Логвин, Б. Боске // - М.: EERC, 2002.- 59 с. 
11. Niskanen A. Economic accessibility of forest resources in Northwest Russia / A. Niskanen, A. Petrov, G Filoushkina // Scientific paper presented in Biennial meeting of the Scandinavian Society of Forest Economics and 3rd BerkeleyKVL Conference of Natural Resource Management May 21-25, 2002, Gilleleje, Denmark 